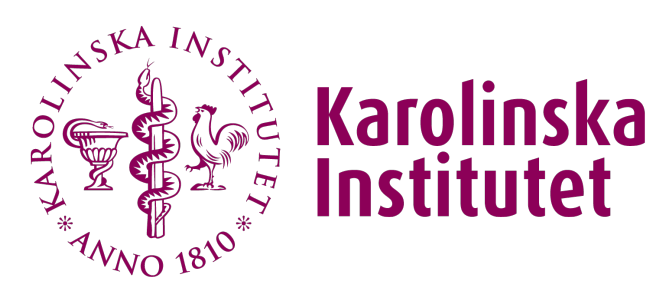

Karolinska Institutet

http://openarchive.ki.se

This is a Peer Reviewed Accepted version of the following article, accepted for publication in Journal of Allergy and Clinical Immunology.

2017-03-13

\title{
Association between parental age and asthma in a population-based register study
}

Almqvist, Catarina; Olsson, Henrik; Ullemar, Vilhelmina; D'Onofrio, Brian M; Frans, Emma; Lundholm, Cecilia

J Allergy Clin Immunol. 2015 Oct;136(4):1103-5.e2.

http://doi.org/10.1016/j.jaci.2015.04.006

http://hdl.handle.net/10616/45585

If not otherwise stated by the Publisher's Terms and conditions, the manuscript is deposited under the terms of the Creative Commons Attribution-NonCommercial-NoDerivatives License (http://creativecommons.org/licenses/by-nc-nd/4.0/), which permits non-commercial re-use, distribution, and reproduction in any medium, provided the original work is properly cited, and is not altered, transformed, or built upon in any way. 


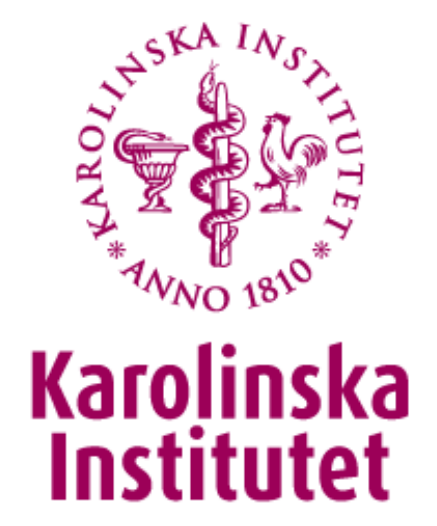

This is an author produced version of a paper accepted by Journal of Allergy and Clinical Immunology. This paper has been peer-reviewed but does not include the final publisher proof-corrections or journal pagination.

Association between parental age and asthma in a population-based register study.

Almqvist C, Olsson H, Ullemar V, D'Onofrio BM, Frans E, Lundholm C.

DOI: https: //dx.doi.org/10.1016/j.jaci.2015.04.006

Access to the published version may require subscription. Published with permission from: Elsevier. 


\section{The association between parental age and asthma in a population-based register study}

Catarina Almqvist MD, $\mathrm{PhD}^{1,2}$

Henrik Olsson $\mathrm{MSc}^{1}$

Vilhelmina Ullemar $\mathrm{BSc}^{1}$

D’Onofrio Brian $\mathrm{M} \mathrm{PhD}^{3}$

Emma Frans $\mathrm{PhD}^{1}$

Cecilia Lundholm $\mathrm{MSc}^{1}$

${ }^{1}$ Department of Medical Epidemiology and Biostatistics, Karolinska Institutet, Stockholm, Sweden

${ }^{2}$ Astrid Lindgren Children's Hospital, Lung and Allergy Unit, Karolinska University Hospital, Stockholm, Sweden

${ }^{3}$ Department of Psychological and Brain Sciences, Indiana University, Bloomington, USA

Corresponding Author:

Catarina Almqvist MD, $\mathrm{PhD}$

Professor, Pediatrician

Department of Medical Epidemiology and Biostatistics

PO Box 281, Karolinska Institutet

SE 17177 Stockholm, SWEDEN

T. +46701160852

E. catarina.almqvist@ki.se

\section{Funding source:}

This work was supported through the Swedish Research Council through grant no. 2011-3060 and the Swedish initiative for Research on Microdata in the Social and Medical Sciences (SIMSAM) framework grant no. 340-2013-5867, the Swedish Heart Lung Foundation, Åke Wiberg foundation, Sällskapet Barnavård, grants provided by the Stockholm County Council (ALF project), the Strategic Research Program in Epidemiology at Karolinska Institutet and the National Institute of Child Health and Human Development. 


\section{Capsule summary}

In a nationwide population-based study with family design, we found an association between decreasing parental age and asthma in early childhood. The effect was independent of familial and potentially confounding factors.

\section{Key words}

Asthma, age, child, confounders, epidemiology, maternal age, paternal age, pediatric 


\section{To the editor,}

Asthma is one of the most common chronic diseases in childhood and adolescence. Risk factors have been sought in utero and the early feto-maternal environment. ${ }^{1}$ Some studies have suggested that children of very young mothers are at increased risk of asthma. ${ }^{2,3}$ Others claim that the risk is not related to age of the mother but rather report bias for health outcomes in firstborns as opposed to younger siblings. ${ }^{4}$ Paternal age as a risk factor for offspring asthma has not yet been studied and could shed additional light on mechanisms related to fetomaternal effects or bias.

Parental age as a risk factor for negative health outcomes has obtained much interest in the area of neuropsychiatric disease, including bipolar disorders and academic morbidity. Although the mechanism behind this association is unknown, a causal link involving de novo mutations, epigenetic alterations and/or fetal programming has been suggested. ${ }^{5}$ There is also recent data to support that the diversity in mutation rate of single nucleotide polymorphisms is dominated by age of the father at conception of the child. ${ }^{6}$ It has been suggested that the negative health outcomes related to parental ages is confounded by parental or family characteristics. By using family designs we can rule out all genetic and environmental factors shared by siblings, half siblings or cousins, and thus strengthen causal inferences. ${ }^{7}$

The aim of this study was to (1) estimate the association between parental age (maternal and paternal respectively) and offspring asthma using family design (half siblings and cousins from mother's sister and father's brother $)^{8}$ and (2) to explore potential confounders, mediators and effect modifiers related to the association.

The Swedish Medical Birth Registry (MBR) was used to establish two cohorts of children, one consisting of children born during July 2005-2009 (early-childhood), and another of 
children born during 1999-2002 (mid-childhood). We excluded children with unknown fathers and multiple births, leading to 445,727 children in the early-childhood cohort and 333,032 children in the mid-childhood cohort.

Asthma was defined using the National Patient Register (NPR) and the Swedish Prescribed Drug Register (SPDR). Asthma in the early-childhood cohort was defined as a diagnosis from NPR combined with dispensed asthma medication. Asthma in the mid-childhood cohort was defined as either a diagnosis from NPR during $7^{\text {th }}$ year of life, or dispensed asthma medication during $7^{\text {th }}$ year of life. ${ }^{9}$

We used parental age both as continuous variables and categorized into 5-year intervals with 25-29 years at birth of offspring as reference category. Continuous age variables per 5-year increases were also used.

For the early-childhood cohort we used Cox regression with attained age as the underlying time scale to estimate hazard ratios (HRs) of the association between parental age and offspring asthma. Due to non-proportional hazards age specific hazard ratios are presented. For the mid-childhood cohort we used logistic regression to estimate prevalence odds ratios (ORs) of the association between parental age and offspring asthma. Robust standard errors were used to account for familial clustering of observations.

We used family designs to account for unmeasured confounding shared within families, with asthma discordant half-siblings/cousins. This was implemented using stratified cox regression and conditional logistic regression with conditioning on half-sibling or cousin pairs.

The study was approved by the Regional Ethical Review board in Stockholm, Sweden. 
Cumulative asthma incidence in the early-childhood cohort was $3.9 \%$ during follow up, while the asthma prevalence in the mid-childhood cohort was $6.3 \%$. Parental age distributions in both cohorts were similar as were other background characteristics of children (sex, birth weight) and parents (parental education, maternal BMI, parity), Table E1 in the Online Repository.

Estimates of age-specific HRs for the early-childhood cohort and ORs for the mid-childhood cohort adjusted for parental education, maternal BMI and parity are presented in Figure 1. During the first year of life the higher maternal and paternal age was associated with lower rates of offspring asthma. This pattern was more pronounced for incidence at age 1-4.5. In the analysis of the mid-childhood cohort, maternal age groups 24 years or younger were associated with lower odds of offspring asthma, while the odds of asthma decreased with higher paternal age.

In analyses using parental age as a continuous measure and family designs to account for unmeasured confounding shared within families, lower rates of offspring asthma with advancing parental age remained in the early childhood cohort after adjustment for confounders, which were statistically significant for asthma discordant cousins (Table 1). In the mid-childhood cohort, the lower odds were stable only for paternal but not maternal age in the cohort analyses. Sub-analyses in both cohorts showed that the results were not driven by the oldest age category.

We found an association between decreasing parental age and asthma in early childhood. This effect was independent of familial and other potentially confounding factors, such as parity and the other parent's age. In mid-childhood there were no consistent associations. 
Previous studies have not accounted for factors shared within families, and are thus comparable primarily to the cohort results in this study, with which the previously seen patterns generally agree for factors shared within families. ${ }^{2,3}$

Strengths of this study are that we had access to population-based data with rich information about covariates, and importantly, information on family relations, allowing several analyses which extend beyond previous publications. Limitations of the family designs include the requirement of exposure- and outcome-discordant sib- or cousin pairs. ${ }^{8}$ When individuals within a family have children together with partners within the same age range exposurediscordance becomes less common and larger populations are needed to reach statistical significance. Family designs allow us to rule out shared environmental factors, but do not tell us which within-family environmental factors were of importance.

In conclusion this study confirms the decreasing risk of early childhood asthma with maternal age, reports that this is also true for paternal age and that the association is independent of shared familial factors. We were not able to identify important mediators and effect modifiers related to the association, which calls for studies to explore mechanisms related to de novo mutations, feto-maternal and early life environment. 


\section{References}

1. Tedner SG, Ortqvist AK, Almqvist C. Fetal growth and risk of childhood asthma and allergic disease. Clin Exp Allergy 2012; 42(10): 1430-47.

2. Laerum BN, Svanes C, Wentzel-Larsen T, et al. Young maternal age at delivery is associated with asthma in adult offspring. Respir Med 2007; 101(7): 1431-8.

3. Metsala J, Kilkkinen A, Kaila M, et al. Perinatal factors and the risk of asthma in childhood--a population-based register study in Finland. Am J Epidemiol 2008; 168(2): 170-8.

4. McKeever TM, Lewis SA, Smith C, et al. Siblings, multiple births, and the incidence of allergic disease: a birth cohort study using the West Midlands general practice research database.

Thorax 2001; 56(10): 758-62.

5. Frans EM, MacCabe JH, Reichenberg A. Advancing paternal age and psychiatric disorders. World Psychiatry 2014, In Press.

6. Kong A, Frigge ML, Masson G, et al. Rate of de novo mutations and the importance of father's age to disease risk. Nature 2012; 488(7412): 471-5.

7. Ortqvist AK, Lundholm C, Kieler H, et al. Antibiotics in fetal and early life and subsequent childhood asthma: nationwide population based study with sibling analysis. BMJ 2014; 349: g6979.

8. D'Onofrio BM, Lahey BB, Turkheimer E, Lichtenstein P. Critical need for familybased, quasi-experimental designs in integrating genetic and social science research. Am J Public Health 2013; 103 Suppl 1: S46-55.

9. $\quad$ Ortqvist AK, Lundholm C, Wettermark B, Ludvigsson JF, Ye W, Almqvist C. Validation of asthma and eczema in population-based Swedish drug and patient registers.

Pharmacoepidemiol Drug Saf 2013; 22(8): 850-60. 


\section{Figure legend.}

Figure 1. Association between parental age and offspring asthma. Hazard ratios (HR) for the early-childhood cohort and odds ratios (OR) for the mid-childhood cohort with associated $95 \%$ confidence intervals using age group 25-29 years as reference category. HRs and ORs of maternal and paternal ages were mutually adjusted for each other, parity, socio-economic factors and maternal BMI in early pregnancy. 